\title{
Research on Techniques of Frequency Source Fusion of Time
}

\author{
GAO Qiang \\ Information Communication Department \\ China Electric Power Research Institute \\ Beijing 100192, China \\ Gaoqiang2010@epri.sgcc.com.cn \\ TENG Ling \\ Information Communication Department \\ China Electric Power Research Institute \\ Beijing 100192, China \\ tengling@epri.sgcc.com.cn \\ WANG Yang \\ Information Communication Department \\ China Electric Power Research Institute \\ Beijing 100192, China
}

\author{
WANG Miaoxin \\ Information Communication Department \\ China Electric Power Research Institute \\ Beijing 100192, China \\ ZHOU Jiakang \\ Telecommunication Company \\ State Grid Jibei Electric Power Co., Ltd \\ Beijing 100053, China \\ DUAN Chengyu \\ Telecommunication Company \\ State Grid Jibei Electric Power Co., Ltd \\ Beijing 100053, China
}

\begin{abstract}
In this paper, we propose a new technique for the fusion of multiple frequency sources. Using the optimal algorithm to select the best reference source from multiple frequency sources, and eliminate the reference source that is deterioration or abnormal. And can be divided into two kinds of methods, such as weight and unequal weight. The test can be divided into two method according to the weight of the available reference source $s$, such as equal weight and unequal weight. The experimental results show that the two methods can correctly remove the abnormal frequency sources, and the fusion results of unequal weight method are better.
\end{abstract}

Keywords-Time and frequency system, Multi-frequency source fusion, Optimization

\section{INTRODUCTION}

This paper researches time-keeping technology of multifrequency source fusion, when referring to atomic fusion algorithm, using a plurality of frequency source integration a more accurate frequency signal for time-keeping. At the same time when work environment deterioration of a frequency source or not working properly ,through self-test mechanisms, the frequency source excluded from the time; keeping group , and then adjust the fusion algorithm, using the remaining frequency sources for time-keeping system. This mechanism can greatly improve the accuracy and reliability of time and frequency unified terminal ${ }^{[1,2]}$.

\section{MULTI-FREQUENCY SOURCE FUSION PRINCIPLE}

The report refer to comprehensive atom algorithm. Design the multi-frequency source fusion algorithm based on the principle and tested analysis. In the atom algorithm,
$\mathrm{N}$ atomic clocks and each reads $h_{\mathrm{i}}(t), \mathrm{i}=1,2 \ldots \mathrm{N}$, comprehensive time scale TA (t), then

$$
\begin{gathered}
T A(t)=\sum_{i=1}^{N} w_{i}(t) h_{i}(t) \\
\sum_{i=1}^{N} w_{i}(t)=1
\end{gathered}
$$

$w_{i}(t)$ is weight of atomic i. When applied to the multifrequency source fusion time; keeping algorithm, frequency fusion can be understood as a plurality of frequency source weighted average summation:

$$
\begin{gathered}
F(t)=\sum_{i=1}^{N} w_{i}(t) f_{i}(t) \\
\sum_{i=1}^{N} w_{i}(t)=1
\end{gathered}
$$

$f_{i}$ represents individual frequency source.

The specific process of the algorithm is described as follows:

a) In the initial state, the amount of data is very small. We can set all frequency source at same status to obtain the average frequency.

b) As data are accumulated and we can calculated current standard deviation and stability of each independent frequency source. 
c) The real-time working state of each individual frequency source were judged according to the standard deviation. When one frequency source is abnormal, it will be removed, and the other frequency source will be repeated by steps a) and b).

d) Calculation of the weights of each frequency source using the stability, and the weighted average frequency can be calculated.

Frequency source is constantly running, accumulating new data, removing old data, repeating steps (b), (c), and adjust the weight of each individual frequency source, update frequency.

With the increase of time, the new data to be accumulated, the old data is removed, repeat steps (b), (c), and constantly adjust the frequency of the independent frequency source, update the fusion frequency.

In summary, two key issues of frequency source fusion algorithm are weight selected and abnormal judged. Experiments were done to solve this two problems.

\section{EXPERIMENTAL VERIFICATION}

\section{A. Effect of weight on fusion frequency}

Typically, there are two methods of determining the weights, equal weight method and unequal weight method. In equal weight method, each individual frequency sources take equal weights. In unequal weight method, according to the stability of each independent frequency source to obtain weights. In atom algorithm, unequal rights are based on the stability of each atomic clock to obtain the weights. Reference to this method, in a multi-frequency source fusion algorithm, count down each individual frequency source stability as weights, the higher the frequency source stability is, the better the weight is. As formula (2) below.

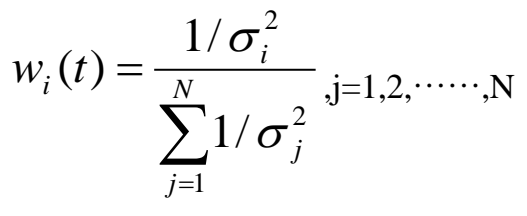

$\sigma_{i}^{2}$ represents Allan variance or standard deviation, no matter what kind of variance, it can make a comprehensive measure of the noise variance is minimized.

In order to obtain the ideal stable fusion frequency, the frequency fusion test is carried out with equal weight method and unequal weight method. This report uses three rubidium atomic frequency standard, and their relative accuracy is $1.2 \mathrm{E}-10,-1.8 \mathrm{E}-10,-1.5 \mathrm{E}-10$. The stability of frequency source is represented by Hadamard ${ }^{[3]}$.

\section{1) Equal weight method}

The algorithm is shown in the flow chart, and the equal weight method is always use the same weight value when calculating the fusion frequency. In this experiment, three independent frequency sources are chosen, so the weight of each frequency source is $1 / 3$. The max deviation of fusion frequency is 3.19E-11, and its stability are shown in figure 1. The abscissa of figure 1 stands for time interval, unit second.

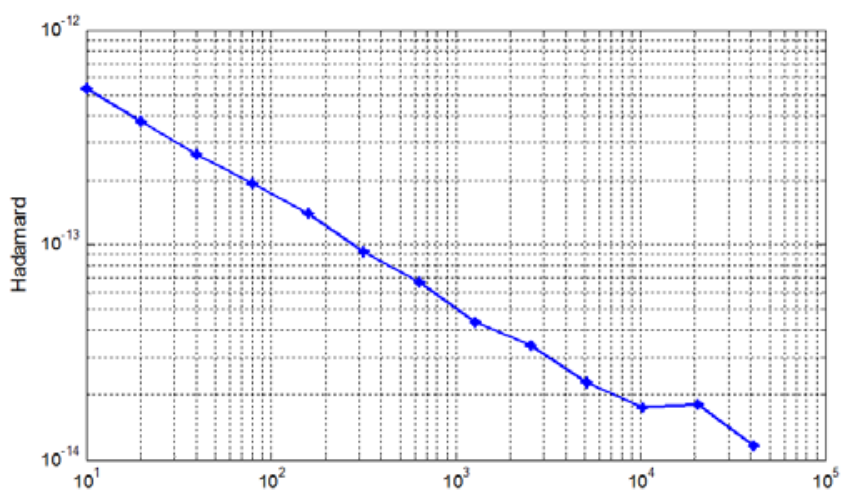

Fig. 1. fusion frequency stability (Equal)

The frequency of equal weight and the stability of the independent frequency source are compared in table 1. It can be seen that the stability of the independent frequency source F1 is the best, F2 and F3 are same, when the interval is greater than 20000s, and the stability of F3 is decreased. After using equal weight method to merge the three frequency sources, F1 does not give full play to its own advantages, the frequency of F2 and F3 has a great influence on the stability of the fusion frequency, resulting in the stability of the fusion frequency is not particularly ideal.

TABLE I. COMPARISON OF STABILITY

\begin{tabular}{|c|c|c|c|c|}
\hline Tau(s) & $\mathbf{f 1}$ & $\mathbf{f} 2$ & $\mathbf{f 3}$ & F-equal \\
\hline $2.00 \mathrm{E}+0$ & $2.38 \mathrm{E}-$ & $7.48 \mathrm{E}-$ & $7.94 \mathrm{E}-$ & $3.76 \mathrm{E}-$ \\
1 & 13 & 13 & 13 & 13 \\
\hline $8.00 \mathrm{E}+0$ & $1.13 \mathrm{E}-$ & $3.80 \mathrm{E}-$ & $4.09 \mathrm{E}-$ & $1.95 \mathrm{E}-$ \\
1 & 13 & 13 & 13 & 13 \\
\hline $1.60 \mathrm{E}+0$ & $9.03 \mathrm{E}-$ & $2.81 \mathrm{E}-$ & $2.85 \mathrm{E}-$ & $1.40 \mathrm{E}-$ \\
2 & 14 & 13 & 13 & 13 \\
\hline $6.40 \mathrm{E}+0$ & $4.54 \mathrm{E}-$ & $1.36 \mathrm{E}-$ & $1.32 \mathrm{E}-$ & $6.73 \mathrm{E}-$ \\
2 & 14 & 13 & 13 & 14 \\
\hline $2.56 \mathrm{E}+0$ & $2.04 \mathrm{E}-$ & $7.28 \mathrm{E}-$ & $6.76 \mathrm{E}-$ & $3.44 \mathrm{E}-$ \\
3 & 14 & 14 & 14 & 14 \\
\hline $1.02 \mathrm{E}+0$ & $1.18 \mathrm{E}-$ & $4.29 \mathrm{E}-$ & $3.70 \mathrm{E}-$ & $1.76 \mathrm{E}-$ \\
4 & 14 & 14 & 14 & 14 \\
\hline $4.10 \mathrm{E}+0$ & $9.53 \mathrm{E}-$ & $2.15 \mathrm{E}-$ & $3.43 \mathrm{E}-$ & $1.16 \mathrm{E}-$ \\
4 & 15 & 14 & 14 & 14 \\
\hline
\end{tabular}

2) Unequal weight method

Through equal power experiment, when the performance of three independent frequency source is different, it is not suitable to take the equal weight method. Therefore, experiments should be carried out with unequal weight method. The fusion frequency and its stability can be 
test also. The stability which is represented by Hadamard is shown in table 2 .

TABLE II. STABILITY COMPARISON

\begin{tabular}{|c|c|c|c|c|}
\hline Tau(s) & $\mathbf{f 1}$ & $\mathbf{f 2}$ & $\mathbf{f 3}$ & $\begin{array}{c}\mathbf{f}- \\
\text { unequal }\end{array}$ \\
\hline $2.00 \mathrm{E}+0$ & $2.38 \mathrm{E}-$ & $7.48 \mathrm{E}-$ & $7.94 \mathrm{E}-$ & $2.59 \mathrm{E}-$ \\
1 & 13 & 13 & 13 & 13 \\
\hline $8.00 \mathrm{E}+0$ & $1.13 \mathrm{E}-$ & $3.80 \mathrm{E}-$ & $4.09 \mathrm{E}-$ & $1.25 \mathrm{E}-$ \\
1 & 13 & 13 & 13 & 13 \\
\hline $1.60 \mathrm{E}+0$ & $9.03 \mathrm{E}-$ & $2.81 \mathrm{E}-$ & $2.85 \mathrm{E}-$ & $9.65 \mathrm{E}-$ \\
2 & 14 & 13 & 13 & 14 \\
\hline $6.40 \mathrm{E}+0$ & $4.54 \mathrm{E}-$ & $1.36 \mathrm{E}-$ & $1.32 \mathrm{E}-$ & $4.86 \mathrm{E}-$ \\
2 & 14 & 13 & 13 & 14 \\
\hline $2.56 \mathrm{E}+0$ & $2.04 \mathrm{E}-$ & $7.28 \mathrm{E}-$ & $6.76 \mathrm{E}-$ & $2.46 \mathrm{E}-$ \\
3 & 14 & 14 & 14 & 14 \\
\hline $1.02 \mathrm{E}+0$ & $1.18 \mathrm{E}-$ & $4.29 \mathrm{E}-$ & $3.70 \mathrm{E}-$ & $1.18 \mathrm{E}-$ \\
4 & 14 & 14 & 14 & 14 \\
\hline $4.10 \mathrm{E}+0$ & $9.53 \mathrm{E}-$ & $2.15 \mathrm{E}-$ & $3.43 \mathrm{E}-$ & $8.80 \mathrm{E}-$ \\
4 & 15 & 14 & 14 & 15 \\
\hline
\end{tabular}

3) Comparison of two kinds of weight selection method

In order to compare the influence of two different weight selection methods on the stability of the fusion frequency, we made a curve in Figure 2. The comparison results show that, in the case of the performance of the independent frequency source, unequal weight average fusion algorithm can obtain more satisfactory results.

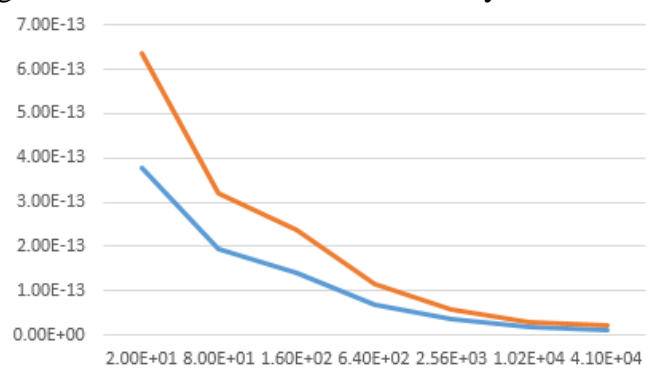

-equal —unequal

Fig. 2. the comparison of two methods

\section{B. Judgment of abnormal frequency source}

In the experiment, the accuracy of the F3 independent frequency source was mutated. The accuracy of the frequency mutate from -2E-11 to +2E-11 in 200000s.

As the algorithm flow is described, the frequency source is judged by the standard deviation of the frequency source in real time. The exception of the F3 makes the standard deviation an obvious mutation in 200000s. The algorithm detected from the beginning of 200000s, the standard deviation of F3 is bigger, until the 200920s increased to 8.2036E-12 that is shown in Figure 3.

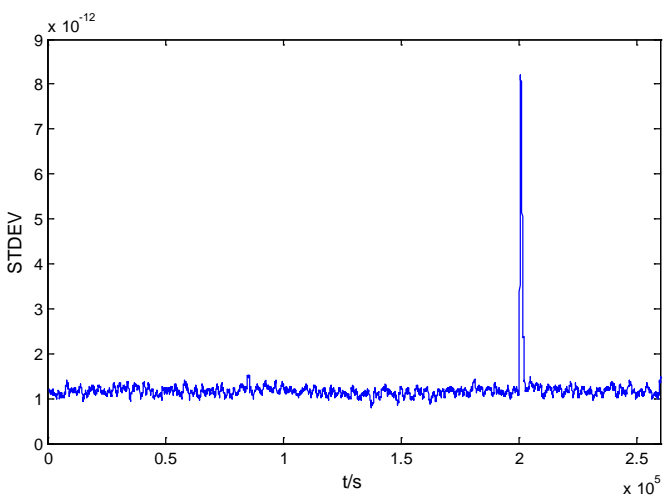

Fig. 3. the change of standard deviation of F3

At the initial stage, the standard deviation is not very obvious, so the algorithm cannot judge the abnormal start time of F3, and until 200042s, the standard deviation increases to $3.03 \mathrm{E}-12$ (normal standard deviation in 1.2E12).The algorithm automatically eliminate frequency source F3, then only use F1 and F2 fusion, the results are shown in the following two. Among them, the max frequency difference of the fusion frequency source is $+9.24 \mathrm{E}-11$, and the stability of the fusion frequency source is shown in Figure 4 . The abscissa of figure 4 stands for time interval, unit second. Because of the delay of the F3 anomaly, the long-term stability of the fusion frequency is affected by a certain degree. If you don't use the fusion frequency of the way, only a single frequency, such as F3, when it appears abnormal it will cause serious consequences. From this point of view, the single frequency obtained by fusion frequency of the fusion algorithm is guaranteed.

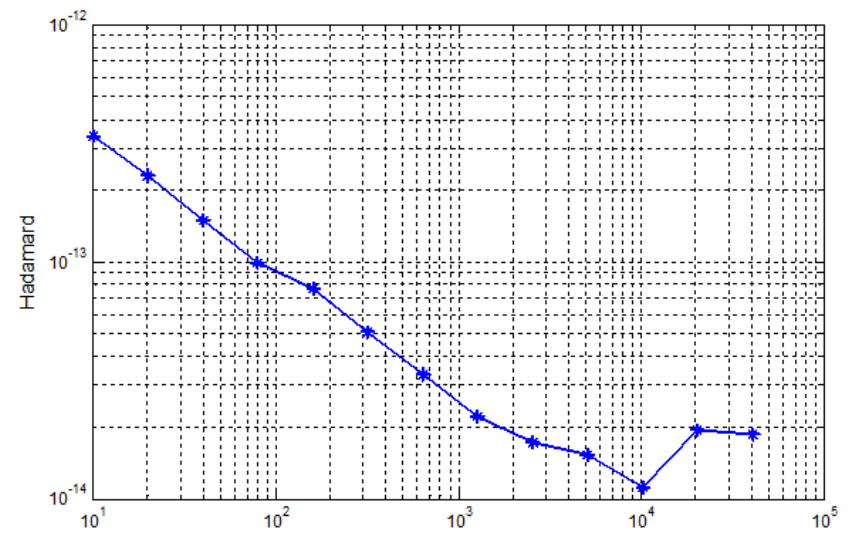

Fig. 4. Stability of fusion frequency source

\section{CONCLUSIONS}

Based on the classical atomic time algorithm, a new algorithm for the fusion of multi-frequency source is proposed and implemented in this system. The results show that when the performance of the independent frequency source is equal, the better fusion frequency can be obtained by equal weighted average method. When the performance of the independent frequency source is different. The average 
performance of the independent frequency source can greatly affect the fusion frequency. At this time, the distribution of unequal power to the high frequency of the performance of high power, low frequency allocation of low frequency can be obtained better fusion frequency. The results of the experiment have positive significance to improve the time performance of the terminal.

\section{ACKNOWLEDGMENT}

This work is supported by the Science and technology project of State Grid Corporation of China (SGCC) "Research and application of the key technology of power synchronization based on time frequency fusion”.

\section{References}

[1] Donald B. Percival. The U. S. Naval Observatory clock time scales [J]. IEEE Trans. Instr. and Meas., 1978, IM-27:376-385.

[2] Stein S. R. Advances in Time-Scale Algorithms[C]. 24th Annual Precise Time and Time Interval (PTTI) Applications and Planning Meeting, 1992, VA, 1-3:289-302.

[3] Gu Yanan, Chen Zhonggui, Shuai Ping. Research on autonomous time synchronization algorithm of navigation constellation based on Hadamard variance [J], Chinese Space Science and Technology,,2010 (1) :1-9. 